\title{
Accelerated Mobile Pages from JavaScript as Accelerator Tool for Web Service on E-Commerce in the E-Business
}

\author{
Agus Wibowo', Guruh Aryotejo ${ }^{2}$, Mufadhol Mufadhol ${ }^{3}$ \\ ${ }^{1,3}$ Departement of Computer System, STEKOM Semarang, Indonesia \\ ${ }^{2}$ Departement of Informatic Management, STEKOM Semarang, Indonesia
}

\begin{tabular}{l} 
Article Info \\
\hline Article history: \\
Received Feb 12, 2018 \\
Revised May 2, 2018 \\
Accepted May 20, 2018 \\
\hline
\end{tabular}

Keyword:

Accelerator

E-business

E-commerce

Javascript

Web service

\begin{abstract}
E-commerce is a sub-part of E-business which includes all kinds of functions and business activities by using electronic data that has the main purpose to increase corporate profits. One of the strategies used is to automate the existing tasks in E-commerce by utilizing web service facilities, which will significantly save time. The use of smartphones as a primary means of information and communication forces web service application providers to improve their services and facilities, such as websites that can be opened quickly and lightly on smartphone devices. This paper will discuss the benefits of accelerated mobile pages as a coding accelerator tool in the javaScript programming language using XML, HTML and XHTML programming as well as involving the use of SOAP, WSDL and NuSOAP that will run on both HTTP and HTTPS protocols. Using accelerated mobile pages as a means of accelerator tool on E-commerce in E-business can directly improve web service performance. This is very prominent when the site is accessed through a smartphone that has limited resources, in the form of website access will feel fast, easy and light.
\end{abstract}

Copyright $\left({ }_{0} 2018\right.$ Institute of Advanced Engineering and Science. All rights reserved.

\section{Corresponding Author:}

Mufadhol Mufadhol,

Departement of Computer System,

STEKOM,

Jl. Majapahit 605, Semarang, Indonesia, phone $+62246723456,+62246710144$.

Email: masyong29@gmail.com

\section{INTRODUCTION}

The rise of the use of smartphones as a primary means of information and communication forces web application service providers to improve their services and facilities [1]. At the beginning of the smartphone was introduced, it can be used to access websites where users no longer need computer equipment as the main tool to access the website, the paradigm shift of website design has to change. Website service providers no longer compete with interesting features and effects in their website pages, but now they have to start thinking not only in the website design but how the look of website services should be opened quickly and lightly on smartphone devices [2]. Smartphones now rule the world and everyone has it. Indonesia is the largest smartphone user in Southeast Asia, where the percentage of internet users through smartphone can be seen in the Figure 1. Indonesia is known as the fourth most ranked country in the world with the percentage of $3.44 \%$ of the world population and the top six for internet world use with ratio reaching $40 \%$ of the total population of Indonesia. This condition is a huge market opportunity for website service providers, it's just how the website service can be accessed by users who have a variety of Internet connections [3], [4].

E-business or Electronic Business as an automated business activity using an information technology or internet technology system enables a company to connect with internal and external data processing systems more efficiently and flexibly [5]. In everyday activities, E-business is often associated 
with the use of E-commerce. E-commerce is more a subdivision of E-business that includes all kinds of functions and business activities by using electronic data that has the main purpose to increase corporate profits. The more people use the internet to get information or shopping online, the greater the company's chances to earn big profits [6]. E-commerce cannot work without a strategy. Strategies in E-commerce are needed to support the company's overall strategic business direction. Convenient of doing business using Ecommerce will be more effective and efficient compared to offline business because the internet does not recognize the boundaries of time, region, culture and the use of its network which can be arranged and controlled as needed [7]. One of the strategies used is to automate the existing tasks in E-commerce by utilizing web service facilities, which will significantly save time [8].

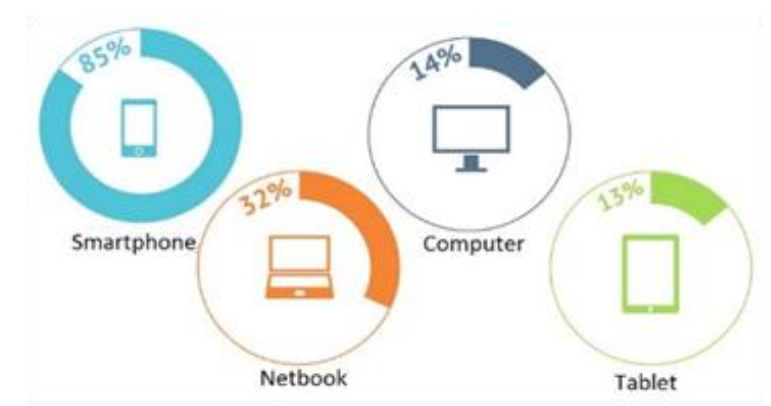

Figure 1. Percentage of internet user tools

Javascript language that is neglected in web programming has evolved into a technology that becomes the backbone of web services with multiplatform technology [9]. Accelerated mobile pages (AMP) is a javaScript-based program, which allows the website to open faster almost without any delay. The rendering process in the background, the optimization of the use of the Preconnect API and the modification of the script layout in accordance with predetermined standards [10] is what makes this possible. The use of AMP programs in web services will benefit smartphone users in the form of fast service access, thereby increasing the convenience of online transactions using smartphones.

\section{RESEARCH METHOD}

This research was conducted using Research and Development method [11] also involving some departments and companies engaged in IT such as e-commerce website service provider.

\subsection{Web service}

Widespread use of technology causes organizations, companies and institutions to compete with technology to achieve their goals. The use of the internet in business is changing from electronic information exchange to business strategy applications, such as marketing, sales and customer service. The use of information technology, especially in the form of websites has made it easier to promote, sell and disseminate information globally [12]. One of the advantages in implementing web applications into E-business is the use of web services in E-commerce [13]. Web service is a self-containing software component and modular selfdescribing application that can be published, allocated, and implemented on the web. Web services are technologies that change the capabilities of internet technology with transactional web features, ie web capabilities to communicate with each other program-to-program (P2P) models. The web focus has been dominated by program-to-user (P2U) communication with business-to-consumer (B2C) interaction, while transactional web tends to program-to-program (P2P) with business-to-business interaction (B2B) [14], [15].

\subsection{Basic development tools}

eXtensible Markup Language (XML) is a markup language that aims to describe the data and the foundation of a web services. The main function of XML is communication between applications, data integration and external application communications with external partners. Simple Object Access Protocol (SOAP) is a protocol for information exchange with decentralized and distributed methods. SOAP is a combination of HTTP and XML, because SOAP generally uses the HTTP protocol as a means of data transport and XML is the protocol for exchange and writing of data. SOAP regulates how the request and response of a web service. Web Services Description Language (WSDL) is an XML-based language used to define a web service and describes how to access the web service. The main function of the WSDL in web 
services is to automate the business-to-business communication mechanisms within the web service via internet protocol. NuSOAP is a collection of PHP classes that allow users to send and receive SOAP messages via the HTTP protocol. NuSOAP is a component-based web service toolkit, and has a base class that provides methods such as variable serialization and SOAP-Envelope packaging. Web service interaction is done with a class client called class "soapclient" and class server called class "soapserver". This class allows users to process the sending and receiving of SOAP messages with the help of several other support classes to complete the process [16].

\subsection{Accelerator tool}

JavaScript is a scripting language based on prototype-based programming concepts. JavaScript names are an implementation of Netscape Communications Corporation for ECMAScript standards. JavaScript is well known for the use of programming languages implemented on the website (as client-side JavaScript) and also used to provide script access for embedded system objects in other applications. JavaScript scripts inserted in HTML or XHTML files running over HTTP or HTTPS protocols must be written between <script> ... </ script> tags [17]. Selecting one website from the search results in Google search engine will make the system perform redirect process from the Google search engine to the destination website. The delay or delay arises during the redirecting process is in a matter of split-second. The goal of the AMP is to reduce the delay. The installation process of AMP is quite easy, as it only installs the AMP plugin as the base foundation or create its own AMP HTML, AMP JS and AMP Cache in javaScript Language. All of these components should be installed, in order to obtain a more complete configuration [18].

\section{RESULTS AND ANALYSIS}

\subsection{E-commerce in the e-business}

E-Commerce is not just a mechanism of selling goods or services through the Internet media, but rather on a business transformation that changes the ways companies work in their daily business activities. Fundamental changes and redefinition of the company's core business often have to be done in connection with this phenomenon, as new paradigms have buried the conventional management principle [19], [20]. The business strategy as well as the results of the evaluation and application portfolio identification formulated in the company's vision, mission and objectives, focuses on cost reduction and maximum utilization of technology in the field of Information Technology. Implementation of Information Technology can be seen from two sides from the point of view of management expansion expectations provided by Information Technology and from the point of view of the implementation and function in the daily operational activities. For example, data transaction security can use network security, while for the physical security of data storage in the room can use room safety sensors [21]. The use of information technology, especially in the web has made it easier in the promotion and spreading of information globally. One form of convenience provided by some web applications is the use of web services that are implemented into E-business [22].

\subsection{Javascript languages programming}

The javaScript programming language is often called the language of the web browser because every internet browser already supports javaScript. The main task of javaScript is to handles user interaction, but after the release of node.js in 2009, the javaScript is no longer limited only to the client side but can also be used on the server or networking side and handle file system access issues. Node.js makes the web service application to be real-time [23].

\subsection{Accelerated mobile pages}

AMP is an open source project from Google based on javaScript programming language and aims to accelerate and improve site performance on mobile users or smartphones. Sites that already use AMP will automatically appear on Google search engine search with additional AMP features in search console on google.

\subsubsection{AMP HTML}

AMP HTML is HTML with some reliable performance changes, which are basically HTML expanded with special AMP properties. The simplest code of the AMP HTML file program in javaScript can be seen in the Figure 2. Although some of the program code or script along with the tags in the AMP HTML page are regular HTML tags, some HTML tags can be replaced with specific AMP tags. This special element is called the AMP HTML component. The AMP page in the website can be found easily on the internet by search engines via HTML tags <link rei = ""> [16]-18], [23]. 


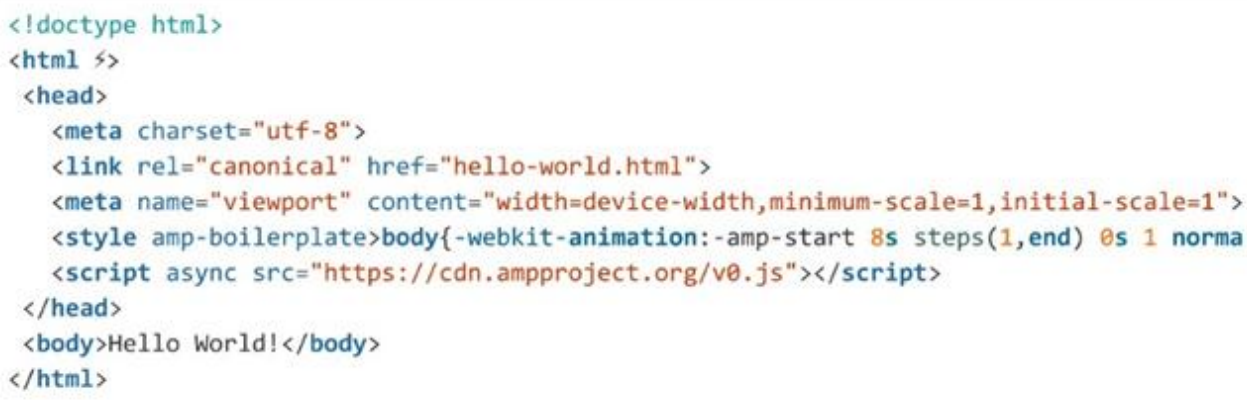

Figure 2. AMP HTML program code

\subsubsection{Library AMP JS}

The AMP JS Library ensures the rendering of AMP HTML pages in the website can be done quickly by implementing all the existing facilities in the AMP into the best performance. The AMP JS Library also manages the loading of content as well as will give a special tag and ensure all the rendering of the website pages is done quickly. The greatest optimization of the AMP JS Library is to make sure everything coming from external content becomes asynchronous, thus none of the activities can block redirecting the pages of the website. In addition, rendering can be optimized through the sandbox process, iframe layout, preliminary calculation of each element's layout on the website page before content is loaded and disabling the slow running CSS selection [18], [23].

\subsubsection{Google AMP Cache}

The Google AMP Cache is a proxy-based website, content calling network, to display all valid AMP documents, which automatically load the AMP HTML pages and place them into the cache. Using Google AMP Cache results in all documents, JS files as well as images loaded from the same source and using HTTP will get maximum efficiency. This cache is also accompanied by a built-in validation system that ensures that the web page is guaranteed to work and not dependent on external content [18]. Google AMP Cache working system can be seen in the Figure 3 .

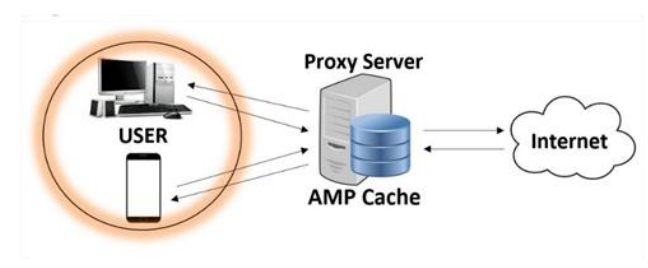

Figure 3. Google AMP cache

Sites that are e-commerce and do not support the native can add amp-iframes for chat and map facilities. Changes in product colors and shapes, sorting and filtering, search results and suggestions, can add an amp-bind facility.

\subsection{Validation and testing AMP process}

The validation system will run a series of statements that will ensure the markup of the website pages matches the AMP HTML specification. Other validator versions are available in one package on every AMP page that can record validation errors directly to the browser console when the web page is rendered. Tests are performed to determine changes and differences after the use of amps on web services [16]-[18], [23].

\subsubsection{AMP validation}

The sites that use AMP must go through the validation process, by entering the domain address and link from the site or the web into the validation system, such as Figure 4. The validation process is completed if the site or e-commerce service is error free and marked with the lightning image. 


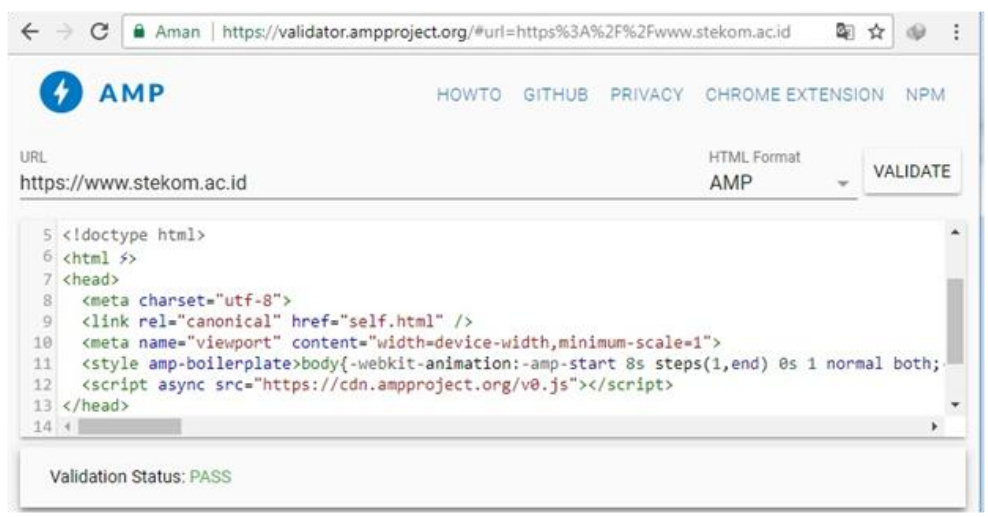

Figure 4. Validation process

\subsubsection{AMP testing}

The changes and differences in rendering on the web service can be performed after the validation process is complete and in the javaScript languages program already have the AMP script "<script async src $="$ https://cdn.ampproject.org/v0.js " > </ script $>$ " installed. The testing process is done by using pingdom tool via mobile device (smartphone and tablet). Performance and time required as well as speed before use of AMP program can be seen in the Figure 5.

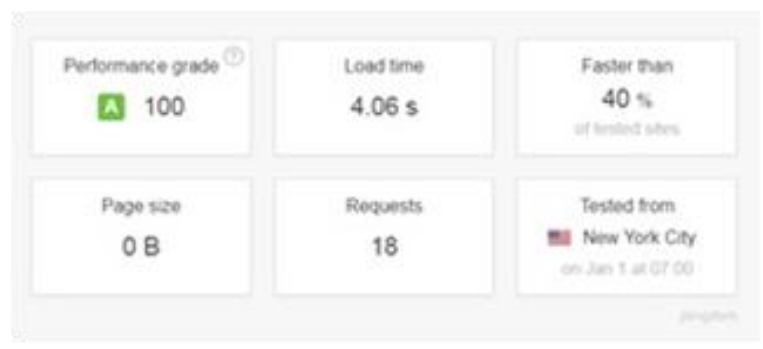

Figure 5. Before AMP installed

There is a speed increase of 1.79 seconds for loading into a web service where loading before using AMP takes 4.06 seconds and after using AMP takes 2.27 seconds. The increase is $25 \%$ in Faster than from $40 \%$ to $65 \%$ with the same performance at 100 performance grade position. This difference is seen in the Figure 6 which shows the improvement process after the use of AMP in javaScript on the web service.

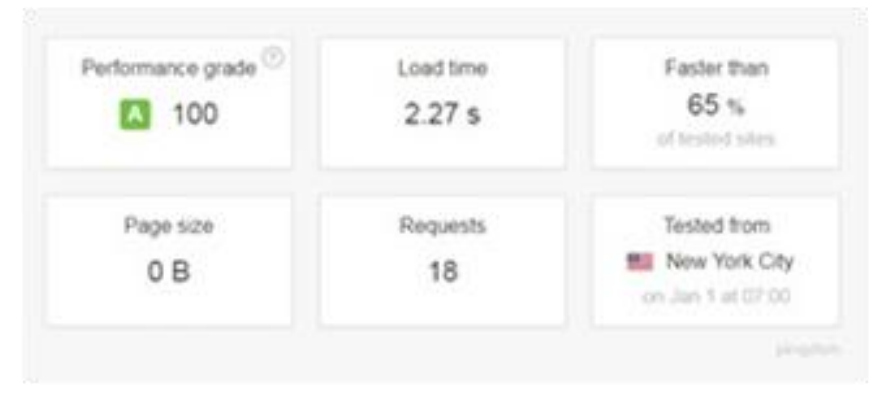

Figure 6. After AMP installed

\section{CONCLUSION}

It is necessary to improve service and quality in the use of web service as a means of utilizing ecommerce to customers. One that should be improved is the speed and lightness of accessing e-commerce 
website services when doing transactions online using a smartphone. JavaScript Programming Languages has developed AMP to be used as an accelerator tool, because of the rendering process in the background, allowing the website to open faster almost without delay. Users will not feel waiting when loading when using a smartphone device, because with this accelerator tool on e-commerce website makes e-commerce activities more attractive because it feels fast and light.

\section{ACKNOWLEDGEMENTS}

This research is supported by Departement of Computer System of Sekolah Tinggi Elektronika dan Komputer (STEKOM) Semarang under the supervision of Research institutions and community service of STEKOM Semarang, Indonesia.

\section{REFERENCES}

[1] S. Y. Lim and S. F. Wong, "Impact of Applying Aggregate Query Processing in Mobile Commerce," International Journal of Business Data Communications and Networking, vol. 8, no. 2, pp. 1-17, 2012.

[2] J. K. Martinsen, H. Grahn and A. Isberg, "Using Speculation to Enhance JavaScript Performance in Web Applications," IEEE Internet Computing, vol. 17, no. 2, pp. 10-19, 2013.

[3] KEMENKOMINFO, "Internet Users in Indonesia Number Six World Rank," Ministry of Communications and Informatics, Jakarta, Indonesia, 2014.

[4] PUSKAKOM, "Internet Users Profile in Indonesia 2015," Association of Indonesian Internet Service Providers (APJII), Jakarta, Indonesia, 2015.

[5] D. K. Gangeshwer, "E-Commerce or Internet Marketing: A Business Review from Indian Context," International Journal of $u$ - and e-Service, Science and Technology, vol. 6, no. 6, pp. 187-194, 2013.

[6] R. Lekha, Nair, D. Sujala and S. D. Shetty, "Streaming Big Data Analysis for Real-Time Sentiment based Targeted Advertising," International Journal of Electrical and Computer Engineering, vol. 7, no. 1, pp. 402-407, 2017.

[7] M. Mufadhol, G. Aryotejo and A. Wibowo, "Netscan and Networx for Management Bandwidth and Traffic with Simple Routing," TELKOMNIKA Telecommunication, Computing, Electronics and Control, vol. 15, no. 1, pp. 464-470, 2017.

[8] T. Zhu, Y. Guo, A. Ju, J. Ma and X. Wang, "An Insider Threat Detection Method Based on Business Process Mining," International Journal of Business Data Communications and Networking, vol. 13, no. 2, pp. 83-86, 2017.

[9] J. Andreeva, I. Dzhunov, E. Karavakis, L. Kokoszkiewicz, M. Nowotka, P. Saiz and D. Tuckett, "Designing and developing portable large-scale JavaScript web applications within the Experiment Dashboard framework," Journal of Physics: Conference Series, 396(052069), pp. 2-11, 2012.

[10] S. Misra and F. Cafer, "Estimating Quality of JavaScript," International Arab Journal Of Information Technology, vol. 9, no. 6, pp. 535-543, 2012.

[11] M. Mufadhol, S. Siswanto, D. D. Susatyono and U. D. Maya, "The Phenomenon of Research and Development Method in Research of Software Engeneering," International Journal of Artificial Intelligece Researh, vol. 1, no. 1, pp. 1-5, 2017.

[12] H. Yang, J. Li, J. Wang and X. Du, "Shortage of logistics for e-commerce in holiday," International Journal of Information Technology and Management, vol. 15, no. 1, pp. 90-98, 2016.

[13] E. V. Pogorelova, I. V. Yakhneeva, A. N. Agafonova and A. O. Prokubovskaya, "Marketing Mix for E-commerce," International Journal of Environmental \& Science Education, vol. 11, no. 14, pp. 6744-6759, 2016.

[14] K. Anusha, C. Yashaswini and S. Manishankar, "Segmentation of Retail Mobile Market Using HMS Algorithm," International Journal of Electrical and Computer Engineering, vol. 6, no. 4, pp. 1818-1827, 2016.

[15] D. Gurram, B. V. Babu and V. Pellakuri, "Issues and Challenges in Advertising on the Web," International Journal of Electrical and Computer Engineering, vol. 4, no. 5, pp. 810-816, 2014.

[16] S. J. S. Bhanu, A. V. Babu and P. Trimurthy, "Implementing Dynamically Evolvable Communication with Embedded Systems through WEB Services," International Journal of Electrical and Computer Engineering, vol. 6, no. 1, pp. 381-398, 2016.

[17] S. Subramaniam, S. C. Haw and P. K. Hoong, "Bridging XML and Relational Databases: An Effective Mapping Scheme based on Persistent," International Journal of Electrical and Computer Engineering, vol. 2, no. 2, pp. 239-246, 2012.

[18] G. Varma, "Google to roll out Accelerated Mobile Pages (AMP) to Main Search Engine Results Pages (SERPs)," Milestone's Galexi® CMS Delivers A Rich AMP Experience, Milestone, Santa Clara, C.A, 2016.

[19] N. M. Hajli, "A study of the impact of social media on consumers," International Journal of Market Research, vol. 56, no. 3, pp. 387-405, 2014.

[20] M. K. Mijinyawa and L. Abdulwahab, "An Extended Framework for Evaluation of Open Source Software Adoption in Small Businesses," Research Journal of Information Technology, vol. 6, no. 4, pp. 248-269, 2014.

[21] M. Mufadhol, G. Aryotejo and D. Y. Kristiyanto, "Rule Based Reasoning Method for Safety Room by Means of Temperature Sensor and Motion Detector," International Journal of Advanced Science Letters, vol. 23, no. 3, pp. 2481-2483, 2017. 
[22] N. Newman, "Journalism, Media, And Technology Trends And Predictions 2017," Digital News Project, Reuters Institute, United Kingdom, 2017.

[23] J. T. Park, H.G Kim and I. Y. Moon, "Design and Analysis of Content Transfer System Using JavaScript and D2D," International Journal of Control Theory and Applications, vol. 9, no. 44, pp. 379-388, 2016.

\section{BIOGRAPHIES OF AUTHORS}

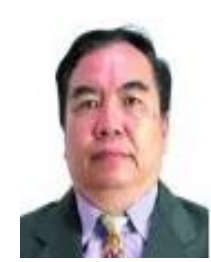

Agus Wibowo (Scopus ID: 57194784814). Department of Computer System, STEKOM Semarang, Semarang Indonesia. Doctoral of Management Program from Diponegoro University, Master of Computer Engineering from Benarif University, Master of Science and Master of Management from Satya Wacana Christian University, Indonesia. Research Interest are in Computer Systems, Software Engineering, Database Management, and Supply Chain Management System.

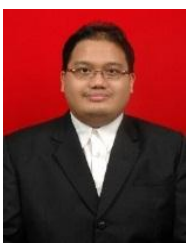

Guruh Aryotejo (Scopus ID: 57194064788). Departement of Informatic Management, STEKOM Semarang, Semarang, Indonesia. Graduates of Computer Science and Informatics from Budi Luhur University, Jakarta, Indonesia and Master of Science from ASIA University, Taichung, Taiwan. Research Interest are in Computer Network, Database Systems, Software Engineering and Information Systems.

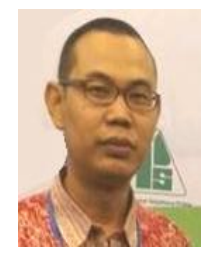

Mufadhol Mufadhol (Scopus ID: 57194073576). Department of Computer System, STEKOM Semarang, Semarang, Indonesia. Graduates of Computer Science and Informatics from STIKUBANK University Semarang, Indonesia and Master of Information System from Diponegoro University, Semarang, Indonesia. Research Interest are in Information Systems, Database System, Artificial Intelligence, Computer Engineering, Software Engineering, and Computer Network. 